\title{
HUBBLE SPACE TELESCOPE PHOTOMETRY OF THE CENTRAL REGIONS OF VIRGO CLUSTER ELLIPTICAL GALAXIES. II. ISOPHOTE SHAPES
}

\author{
FRANK C. VAN DEN BOSCH \\ Leiden Observatory, Leiden, The Netherlands \\ Electronic mail: vdbosch@strw.leidenuniv.nl \\ LAURA FERRARESE \\ Johns Hopkins University, Space Telescope Science Institute, Baltimore, Maryland 21218 \\ Electronic mail: ferrares@jhufos.pha.jhu.edu \\ WALTER JAFFE \\ Leiden Observatory, Leiden, The Netherlands \\ Electronic mail: jaffe@strw.leidenuniv.nl \\ HOLLAND C. FORD \\ Johns Hopkins University, Space Telescope Science Institute, Baltimore, Maryland 21218 \\ Electronic mail: ford@jhufos.pha.jhu.edu \\ ROBERT W. O'CONNELL \\ University of Virginia, Charlottesville, Virginia 22903 \\ Electronic mail: rwo@perseus.astro.virginia.edu \\ Received 1994 March 9; revised 1994 June 17
}

\begin{abstract}
The isophotal shapes of a magnitude limited sample of Virgo ellipticals are presented. These are derived from high resolution $H S T$ photometry. The absence of atmospheric seeing and accurate knowledge of the PSF allows us to perform an accurate deconvolution. Model galaxies were constructed to test the deconvolution algorithms used, and showed that we can accurately recover isophotal shape parameters down to 0.5 . From the isophotal parameters we can classify the galaxies in two subsamples: disky and non-disky galaxies. In three of these disky galaxies we found evidence for a nuclear stellar disk in the inner 1.5. In addition these galaxies also have an outer disk, that seems to break up inside $2^{\prime \prime}-3$. In the two galaxies for which there is kinematic evidence from the literature of a decoupled core, we found no indication for such subsystem from the isophotal shape analysis. In $80 \%$ of these early type galaxies there are indications for dust. For eight of these galaxies the dust has not been detected before.
\end{abstract}

\section{INTRODUCTION}

It has become evident in recent years that the central regions of elliptical galaxies are far from simple, violently relaxed, isothermal regions, and that important information on the central mass distribution and formation history can be derived from careful photometry of these regions. In Paper I of this series (Jaffe et al. 1994b) we describe the need for $H S T$ observations of elliptical nuclei, the selection of a complete sample of 14 bright Virgo cluster ellipticals, and the observing and calibration procedures. The reduced images are also presented there. In this paper we present a study of the isophotal shapes of the inner kpc of the sample galaxies, whereas Paper III (Ferrarese et al. 1994) deals with the luminosity profiles.

Previous studies of the isophotes of elliptical galaxies have shown that the isophotal shapes of elliptical galaxies provide important information. For example, the isophote twists that are observed in many galaxies are a strong indication for a triaxial potential or for tidal effects from a massive, nearby companion (e.g., Mihalas \& Binney 1981; di Tullio 1979). Furthermore, in the last few years it became evident that isophote shapes of ellipticals show surprisingly good correlations with a variety of other properties of these objects, such as radio and $\mathrm{x}$-ray fluxes, absolute magnitude and angular momentum, dividing the ellipticals into two families; the boxy and the disky ellipticals (cf. Bender 1988). The boxy ellipticals are the more luminous galaxies, and often show nuclear activity. In addition, their systematic velocities are small, while velocity dispersions can be very high, implying that boxy ellipticals are pressure supported. In contrast, ellipticals with pointed isophotes show low velocity dispersion and high rotation velocities, probably as the result of the presence of a weak stellar disk embedded in the spheroidal. The strong similarity between these "disky" ellipticals and S0s suggests that the former represent the extreme case of S0s with vanishing faint disks in the continuation of the disk-to-bulge sequence. A complication here is that there is no a priori reason why the isophotes of the spheroids themselves should be perfectly elliptical. It has been shown (e.g., Miyamoto \& Nagai 1975; de Zeeuw et al. 1986) that one-component mass models in axisymmetric or 
triaxial potentials can also produce pointed isophotes. Disky isophotes may therefore not always indicate the presence of an embedded disk, and one normally needs additional (kinematical) data to be confident about the presence of a disk component.

Our chief goal is to investigate the morphological features in the central regions of ellipticals and to see if they are distinct from their outer parts. Although previous isophotal studies of the central regions in elliptical galaxies (Bender 1988; Nieto et al. 1991a) were severely limited by the effects of seeing, they revealed that many ellipticals show strong changes in ellipticity and/or position angle. In several cases it was found that the isophotes became pointed, suggesting the presence of a nuclear stellar disk. In many cases, changes in the isophotal shapes in the central region of ellipticals were associated with so called "decoupled cores." We will refrain from this terminology, because the interpretation of these changes is far from unambiguous. In this study, we examine the nature and frequency of these "photometrically distinct nuclei."

The outline of this paper is as follows. In Sec. 2 we discuss the observations and the reduction procedure. Section 3 describes the tests used to study the accuracy of the isophotal analysis and presents the results on the individual galaxies. Finally, in Sec. 4, we discuss the overall results and the correlations found.

\section{OBSERVATIONS AND REDUCTION PROCEDURE}

The sample, observation parameters, and reduction procedure are described in Paper I. The sample consists of 14 of the 17 brightest galaxies in the Virgo cluster classified as either E or E/S0 in the Revised Shapley-Ames Catalogue of Bright Galaxies (Sandage \& Tammann 1981; hereafter referred to as RSA). The other 3 could not be observed with $H S T$ for lack of guide stars. The image of M87, that we extracted from the public $H S T$ archive (see Paper I), has a very low signal-to-noise ratio. We were just able to determine the luminosity profile from this image (see Paper III), but the higher order isophotal shape parameters could not be accurately determined. Furthermore, the presence of the nonthermal point source, the optical jet, and the $\mathrm{H} \alpha$ filaments, which were shown to contain dust (Sparks et al. 1993; Zeilinger et al. 1993), make the interpretation of the isophotal parameters very complicated. We therefore decided to delete M87 from our sample.

\subsection{Deconvolution}

All images have been deconvolved in order to correct for the spherical aberration of the HST optics. Since we want to feel confident about the results of the deconvolution applied, we have compared two different reconstruction methods: the Richardson-Lucy (RL) algorithm (Lucy 1974; Snyder 1990), as implemented in the STSDAS package in IRAF, and a Fourier filtering algorithm. The advantage of the RL-method over Fourier filtering is that it is forced to be positive and the flux is conserved both globally and locally at each iteration. On the other hand the RL-deconvolution is a nonlinear algorithm whose reliability is difficult to estimate. We therefore

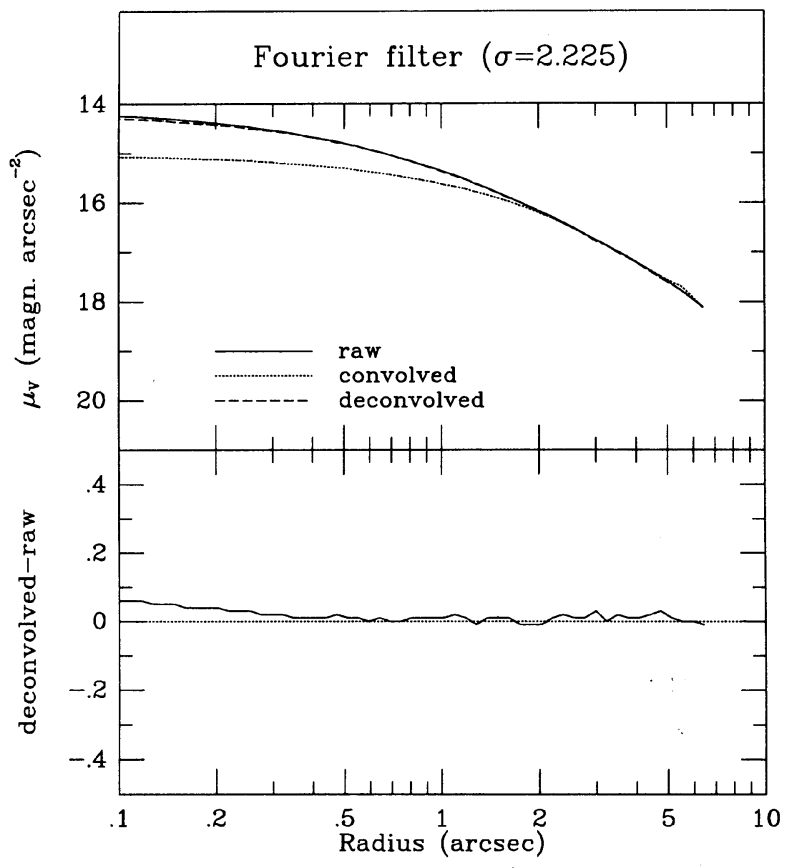

FIG. 1. The upper panel shows the luminosity profiles for a model galaxy. The raw model (solid line) has a Hubble profile. The dotted line shows the luminosity profile of this galaxy after convolution with the HST PSF. After Fourier filtering of this convolved model with $\sigma=2.225$ cycles/arcsec, the luminosity profile (dashed line) very much resembles the raw one. The lower panel shows the difference between the raw and deconvolved luminosity profiles.

also Fourier filtered the raw images in order to compare the differently restored images, and to get an idea of the credibility of the deconvolved images. The Fourier filtering is described by the formula:

$$
S=\hat{F}\left(\frac{\hat{F}(I)}{\hat{F}(K)} \times e^{-r^{2} / 2 \sigma^{2}}\right) .
$$

Here $I$ is the HST image, $K$ the HST PSF, and $S$ the deconvolved image. The operator $\hat{F}$ denotes the Fast Fourier Transformation. The exponential describes a high frequency cutoff used to limit the noise growth. We tested this Fourier filtering for different values of $\sigma$ by comparing the luminosity profile of a input model galaxy with the deconvolved image $S$. For small values of $\sigma$ the deconvolved luminosity is systematically lower than for the raw (unconvolved) model at small radii, whereas the deconvolved image $S$ becomes very noisy for large values of $\sigma$. After extensive testing (also by studying the isophotes, see Sec. 3.1) we settled for $\sigma=2.225$ cycles/arcsec. Figure 1 shows that, for this value, the difference between the raw model, having a modified Hubble luminosity profile, and $S$ is smaller than 0.1 mag $\operatorname{arcsec}^{-2}$, for $r \geqslant 0$ ". 1 . In Paper III it is shown that convergence for the RL-algorithm is achieved after 100 iterations, by testing the RL deconvolution using similar tests as the ones described here. Both the Lucy and the Fourier deconvolved images suffer from boundary problems. Therefore the outer 50 lines and columns are removed, after deconvolution on the entire $800 \times 800$ PC 6 CCD. 


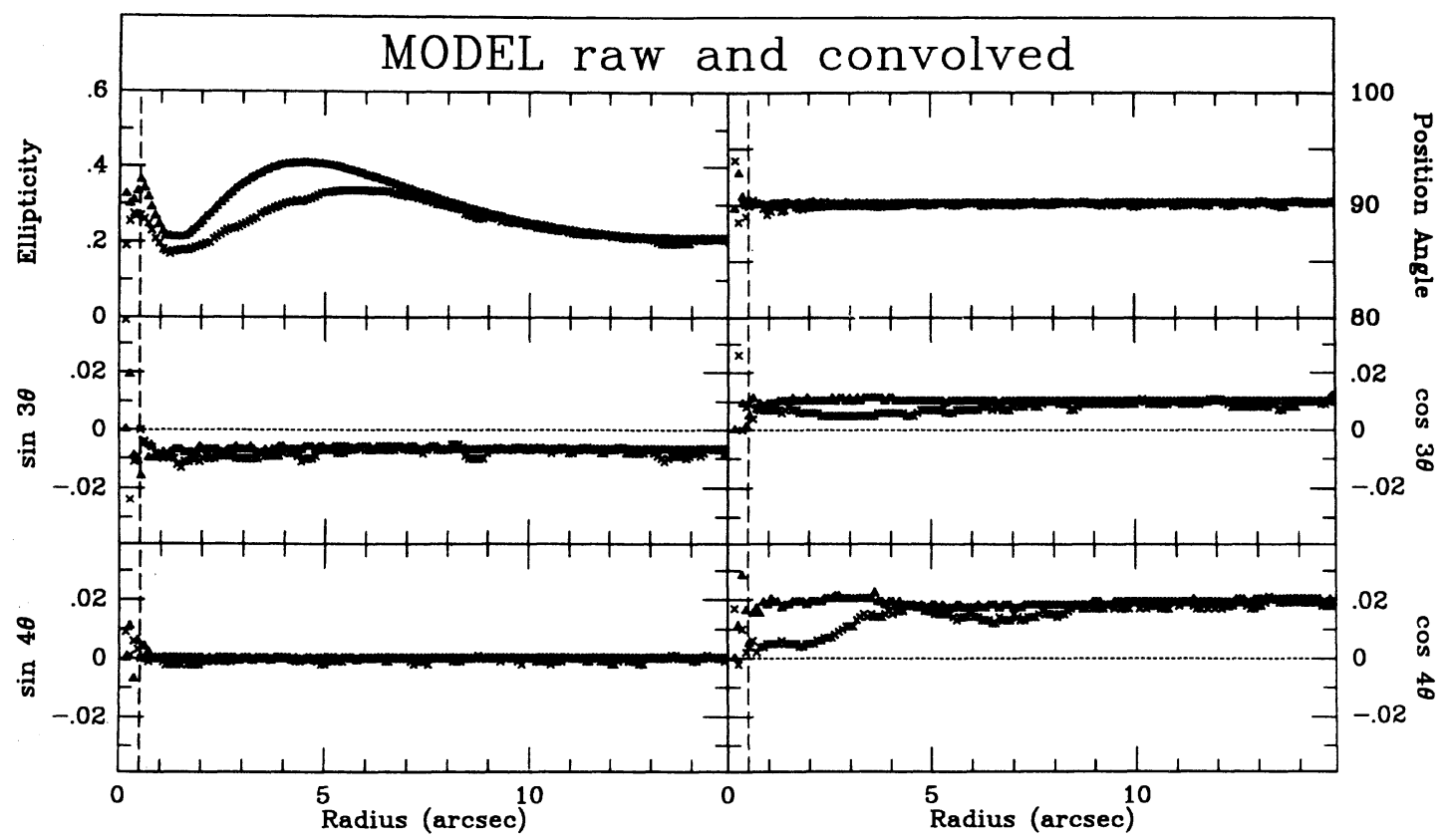

FIG. 2. Isophotal parameters of a model-galaxy before and after convolution with an $H S T$ model-PSF and addition of photon-noise.

\section{ISOPHOTAL SHAPE ANALYSIS}

\subsection{Parameter Extraction}

For each of our galaxies we fit a series of elliptical isophotes to the deconvolved surface brightness. The ellipticity and position angle profiles are found at each semimajor axis length, using the isophote fitting program ELLIPSE in the STSDAS package of IRAF. The program uses an iterative method described by Jedrzejewski (1987). During each iteration the center of the isophote, ellipticity and position angle are allowed to vary. If, after a certain number of iterations, a satisfactory fit is found, the $\sin$ and $\cos 3 \theta$ and $4 \theta$ terms are then derived that describe the high-order deviations of the isophotes from pure ellipses by means of the following Fourier expansion:

$$
\frac{\delta r(\theta)}{r(\theta)}=\sum_{n=3}^{4}\left[A_{n} \sin (n \theta)+B_{n} \cos (n \theta)\right] .
$$

For a perfect ellipse these coefficients are all equal to zero. Typically, the amplitudes $A_{n}$ and $B_{n}$ are small, rarely exceeding 0.02 . Note that positive $\cos (4 \theta)$ terms correspond to "disky" isophotes, whereas "boxy" isophotes give rise to negative values of $B_{4}$.

\subsection{Testing Deconvolution Techniques on Simulated Galaxies}

In order to investigate the accuracy of the ellipse-fitting program and to see whether our deconvolution methods induce strange artifacts in the derived brightness distribution, we have simulated the reduction process on model galaxies having a variety of ellipticity and position angle profiles. The model-galaxies had a modified Hubble profile along the major axis $r$

$$
\Sigma(r)=\frac{\Sigma_{0}}{\left(1+r / r_{0}\right)^{2}},
$$

with $r_{0}=2$ ".2. Some models had pure elliptical isophotes, while others were constructed having nonzero $A_{n}$ and $B_{n}$ so as to test the ability of the program to detect deviations from perfect ellipticity. After construction of each model, it was convolved with a $H S T$ model PSF, and photon noise was added. Consequently, it was deconvolved using in turn the RL-algorithm and the Fourier filtering method, and subjected to the above described isophote fitting procedure.

We discuss here extensively a model-galaxy having an ellipticity profile

$$
\epsilon(r)=0.3-0.1 * \sin \left[2 \pi \log _{10}(r)-\frac{\pi}{2}\right],
$$

where $r$ is the semimajor axis length. The position angle was taken to be constant $\left(\theta=90^{\circ}\right)$, and the isophotes deviate from perfect ellipses as given by Eq. (2) with $A_{3}=-0.007$, $B_{3}=0.01, A_{4}=0.0$, and $B_{4}=0.02$. The central brightness of this galaxy was given a value typical for our sample. In the following we will refer to the noiseless, unconvolved model galaxy as the "raw model," the model convolved with the PSF and with noise added as the "convolved model," and the convolved model after deconvolution as the "restored model."

Figure 2 shows the derived isophotal parameters from both the raw model (filled triangles) and the convolved model (crosses). As can clearly be seen, the ellipticity of the convolved model is systematically lower than that of the raw 


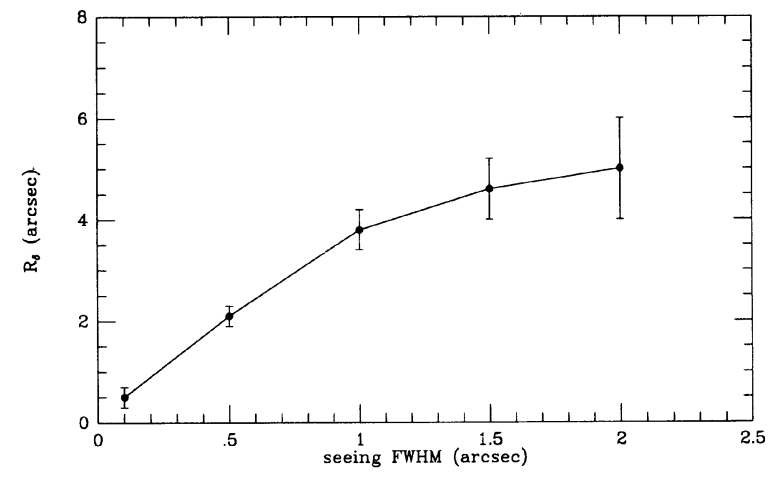

FIG. 3. Influence of seeing on the higher order terms that express deviations from perfect ellipticity, as a function of the FWHM of the (Gaussian) seeing. $R_{\delta}$ is the radius (in arcsec) where the error in the $B_{4}$ term due to smearing becomes larger than $10 \%$.

model for small radii. This is due to the smearing of the central luminosity over a larger area by the round PSF. This effect was previously noted and accurately modeled by Peletier et al. (1990), who found that seeing affects the luminosity distribution out to radii as large as 5-10 times the seeing radius. This was confirmed by our modeling. The ellipticity of the convolved model in Fig. 2 is significantly smaller than the intrinsic value for all radii smaller than $\sim 7^{\prime \prime}$. The PSF of the HST has a sharp $\left(\sim 0^{\prime \prime} 1\right)$ core with a halo extending to a radius of more than $3^{\prime \prime}$. Although the FWHM is $\sim 0$." 1 , only about $16 \%$ of the light from a point source is concentrated inside this radius (Burrows et al. 1991). Obviously, the extended wings of the PSF of the aberrated HST affect the luminosity distribution even beyond radii 10 times the FWHM of the PSF.

Figure 2 also shows that the $4 \theta$ "disky-boxy" parameters are also underestimated out to many times the FWHM convolving size. This effect presumably also exists in groundbased observations. To test this we convolved our models with Gaussians for different values of the FWHM. In Fig. 3 we plot the radius $R_{\delta}$ at which the errors in the $B_{4}$ parameter due to seeing become $10 \%$, as a function of FWHM. The magnitude of the effect confirms that ground-based studies may severely underestimate the diskiness in the central regions.

After deconvolution the isophotal parameters derived from the restored image closely resemble the intrinsic parameters. The differences between the intrinsic parameters and those from the Fourier restored image are shown in Fig. 4 on an expanded scale. Outside 0.5 there are no systematic differences between the intrinsic and the restored values.

Figure 4 also shows two shortcomings of our procedure. First, for the $3 \theta$ and $4 \theta$ parameters the variations caused by the photon noise exceed the values found in most galaxies (e.g., Bender et al. 1988; Franx et al. 1989b; Peletier et al. 1990). Secondly, the isophote fitting algorithm fails at radii $\gtrsim 5$ " because the slope of the luminosity profile per measured pixel is too small [see Jedrzejewski (1987) for details on this criterion]. To solve both of these problems we rebinned the image into larger pixels by averaging over $16(4 \times 4)$ of the original pixels. This increases the per pixel slope of the luminosity profile so that we can extend our analysis out to $15^{\prime \prime}-20^{\prime \prime}$ and also increases the signal-to-noise ratio. This can clearly be seen by comparing Fig. 4 to Fig. 5(a), where we

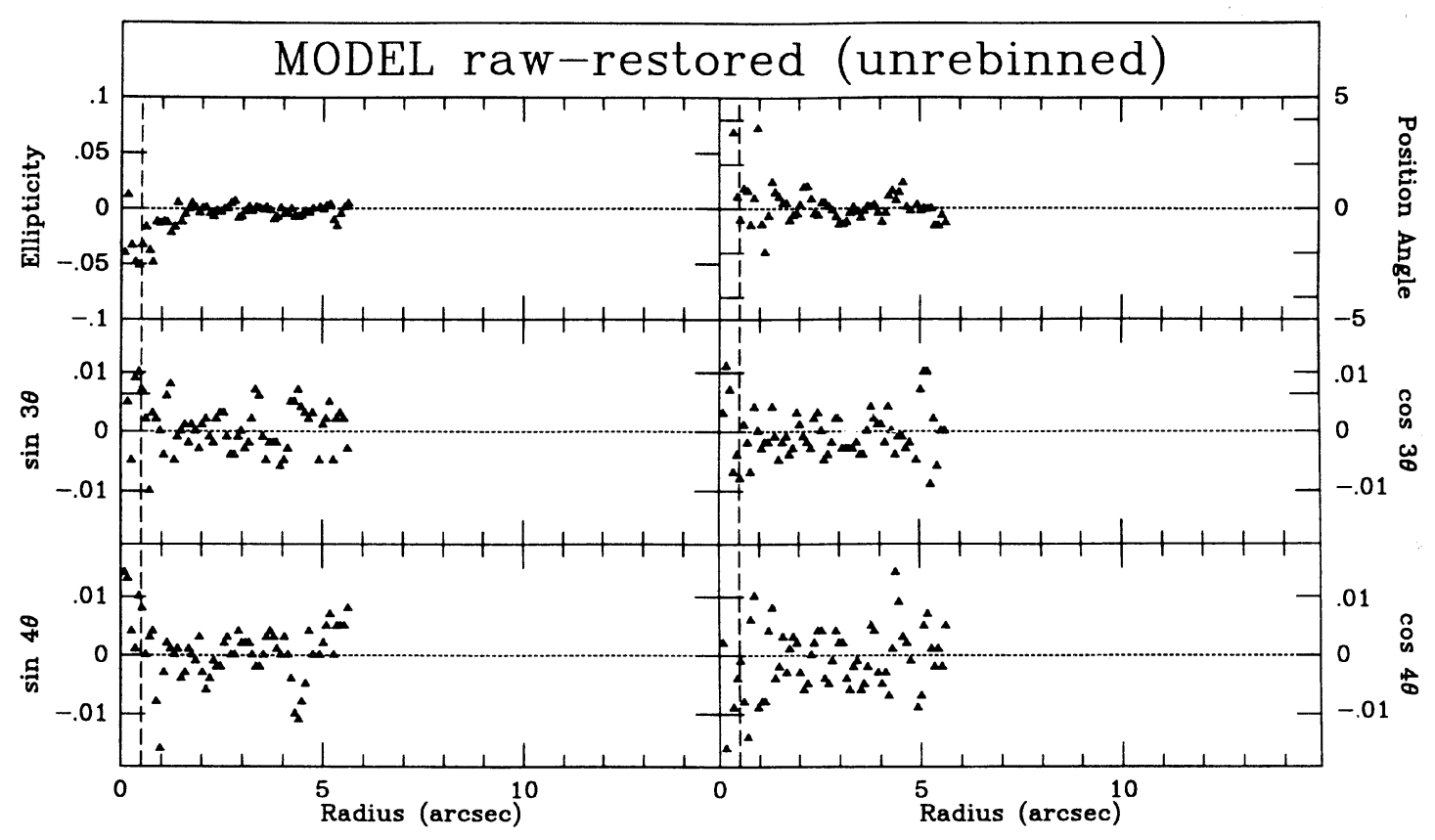

FIG. 4. Difference in the isophotal parameters between those of a raw model-galaxy and those of the same galaxy after convolution, noise addition, and deconvolution by means of Fourier filtering. For radii larger than 5.5 no isophotes could be fitted, since the slope of the luminosity profile is too small. Note the relative large amount of scatter on the higher order terms. 

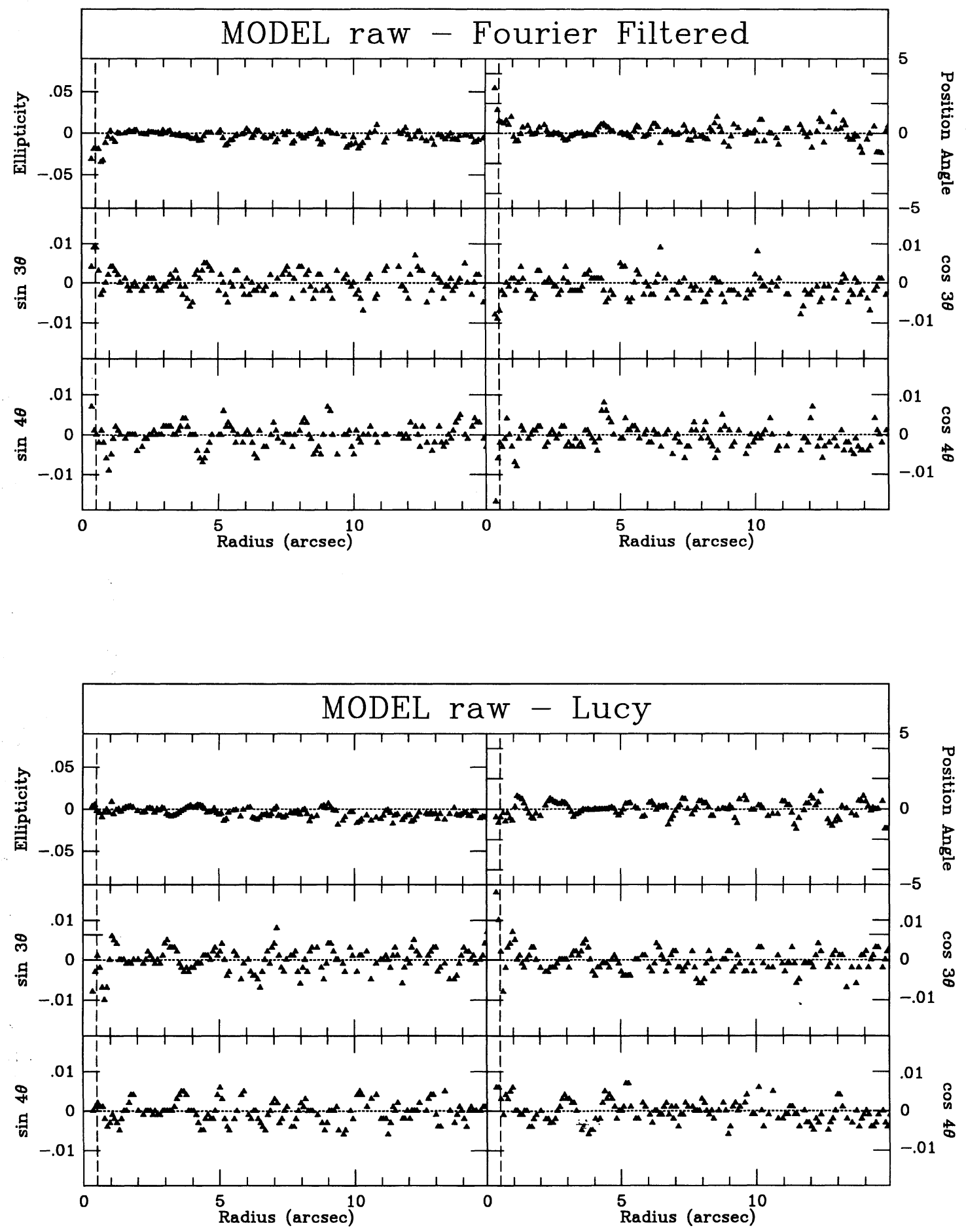

FIG. 5. (a) Same as in Fig. 4, except that the image has been rebinned; each new pixel represents the average value of a $4 \times 4$ matrix of old pixels. Note the reduction in the noise in the higher order terms. (b) Same as (a), except that the convolved model has been deconvolved using 100 iterations of the Richardson-Lucy algorithm instead of Fourier filtering. Note the strong resemblance to (a).

have plotted the differences between the intrinsic parameters and those derived from the rebinned Fourier filtered image.

For all input models the isophotal parameters are not significantly different from the input raw values, for $r \geq 0$ ". 5 . The errors for radii smaller than 0.5 are due to the imperfect deconvolution on these small scales (see also Fig. 1), and to the fact that at semimajor axis lengths of less than 20 pixels, the program uses a bilinear interpolation scheme to estimate the intensity of the galaxy at points intermediate between pixels. As was already pointed out by Jedrzejewski (1987) 
this interpolation has a few unrealistic properties. Our test showed in fact that for $r<0$." 5 the isophotal parameters determined from nonrebinned images were no better than those determined from the rebinned images. We therefore decided to neglect the isophotal parameters for semimajor axis lengths smaller than 0.5. This minimal semimajor axis is shown as a dashed line in Figs. 2, 4, 5, and 6.

Figure 5(b) shows the same data as in Fig. 5(a) but determined from a model restored with the RL-algorithm after 100 iterations. To the noise limit the results are identical, as was the case for all the other models we investigated.

\subsection{Final Method}

After a careful study of models with various isophotal shapes and luminosity profiles we decided to use the $4 \times 4$ rebinned images for the determination of the isophotal parameters. Semimajor axis lengths smaller than 0.5 are excluded as are values where more than $40 \%$ of the isophote lies outside the frame. Bad pixels are flagged and excluded in the isophotal analysis. If a bright star is present in the area studied, a square area covering the entire star is flagged, so that this part of the image is also excluded. Furthermore, at each isophote the brightest $5 \%$ of the pixels at the intensity interval concerned is clipped out of the sample. In this way cosmic rays that were not found by the algorithm used, weak stars and globulars are neglected when trying to fit an ellipse to the isophote. The number of iterations needed to fit an isophote is taken to be between 8 and 80 . If, after 80 iterations, no satisfactory fit is found, the isophote is excluded from further analysis and the next isophote is fitted.

\subsection{Results for Individual Galaxies}

Figure 6 presents the isophotal parameters of the 13 galaxies in our sample. While we present the deviations from pure elliptical isophotes by the amplitudes $A_{n}$ and $B_{n}$ of the $\cos$ and $\sin 3 \theta$ and $4 \theta$ terms, a useful method to study the cause of nonzero sin and $\cos 3 \theta$ and $4 \theta$ terms is by constructing residual maps (see Capaccioli et al. 1987). Such a residual map is constructed by subtracting a model galaxy from the galaxy image concerned. The model is based on the surface photometry of the deconvolved image under the assumption that the galaxy has perfectly elliptical isophotes. The luminosity, ellipticity, and position angle profiles of the model are similar to the real image, as are the centers of the isophotes. Therefore, the residual map shows the residual structure of all terms of order three and higher.

Since our F555W filter passes $\mathrm{H} \alpha+[\mathrm{N}$ II $](\lambda \approx 6600 \AA)$ at $\sim 15 \%$ transmission efficiency, strong emission from ionized gas present in the central regions of the galaxies might have a significant effect on the isophotal shapes. Given the transmission characteristics of the F555W filter, we estimate that for a typical survey galaxy, a cloud of ionized gas of size $\sim 1^{\prime \prime}$ at $3^{\prime \prime}$ from the center, with a flux of $10^{-13} \mathrm{erg} \mathrm{cm}^{-2} \mathrm{~s}^{-1}$ in $\mathrm{H} \alpha+[\mathrm{N}$ II] line emission would cause amplitudes of the 3 and $4 \theta$ terms of the order of $0.01-0.02$. This value of the line emission is typical for active galaxies, and one has to keep in mind that deviations from perfect ellipticity could be due to ionized gas.
As mentioned in Sec. 1, and as will become evident in the following, every galaxy in our sample has a unique morphology in its inner $10^{\prime \prime}$. We will therefore first discuss the results per individual galaxy, and then consider the systematic properties of the whole sample. In Table 1 we list the tabular values derived from the isophotal shape analysis as derived from the Lucy deconvolved images. We note that these values were not significantly different from those derived from the Fourier filtered images. In Fig. 6(n) we show the difference of the isophotal shape parameters derived from the Lucy deconvolved image when compared to the Fourier filtered image of NGC 4342 . Note that the similarity is extremely good even though the isophotal structure of this galaxy is very complex. The consistency of the isophotal shape parameters derived between the Lucy deconvolved and Fourier filtered images is equally good for the other galaxies. Therefore, in Figs. 6(a) $-6(\mathrm{~m})$ we only present the parameters derived from the Lucy deconvolved images.

$$
\text { 3.4.1 NGC } 4472 \text { (M49) }
$$

NGC 4472 is the brightest elliptical in Virgo. The isophotes are well approximated by ellipses from 0.5 to $15^{\prime \prime}$. The galaxy becomes gradually rounder toward smaller radii. Inside $\sim 2^{\prime \prime}$ the isophotal parameters are poorly determined because of the low slope of the luminosity profile, the low ellipticity, and a small patch of dust which disturbs the isophotes. The isophotal profiles show no evidence for a photometrically distinct nucleus whereas Davies \& Birkinshaw (1988) and Franx et al. (1989a) find clear evidence for a kinematically decoupled core in the inner $5^{\prime \prime}$.

\subsubsection{NGC $4374(M 84=3 C 272.1)$}

The giant elliptical radio galaxy, NGC 4374 forms a pair with NGC 4406 (=M86). The outskirts of these galaxies overlap at $\mu_{B}=26$. NGC 4374 harbors dust lanes in its central region (e.g., Hansen et al. 1985; Paper I). These lanes are oriented perpendicular to the radio jet (Hansen et al. 1985). Furthermore, the nuclear region shows a $\mathrm{H} \alpha+[\mathrm{N}$ II $]$ emission line region aligned with the dust lanes, and extending $12^{\prime \prime}$ on both sides of the nucleus (Baum et al. 1988). These features heavily disturb the isophotes for $a \leqslant 10^{\prime \prime}$. At larger radii the complex isophotal shapes are consistent with previous studies of this galaxy (Bender et al. 1988; Peletier et al. 1990).

\subsubsection{NGC 4365}

NGC 4365 is a radio quiet giant elliptical. Surma (1993) found clear evidence for two kinematically distinct components within this object: a hot "bulge"-like component plus a cold "disk"-like subsystem. Surma finds the $\cos 4 \theta$ term to become positive ("disky") inside $\sim 4$ ", consistent with our results. However, the isophotes become boxy again at $a<1$ ". 2 , where Surma's data is unreliable. The ellipticity and position angle remain rather constant over the entire radial range, while the isophotes are almost perfectly elliptical (except for the small positive $\cos 4 \theta$-bump around $2^{\prime \prime}-3^{\prime \prime}$ ). While we found no dust by visual inspection, this galaxy is a far-infrared emitter (cf. Paper I) and a small amount of dust 
may influence the $3 \theta$ and $4 \theta$ components at small radii. Additionally the bumpy profiles might also originate from weak $\mathrm{H} \alpha+[\mathrm{N}$ II] emission detected by Kennicut \& Kent (1983). We therefore argue that there is no conclusive photometric evidence for a decoupled core.
3.4.4 NGC 4473

NGC 4473 is a highly elongated elliptical classified E5 in both the RSA and the Second Reference Catalogue of Bright Galaxies (de Vaucouleurs et al. 1976, hereafter referred to as RC2). The ellipticity slowly increases from 0.36 at $a=15^{\prime \prime}$.
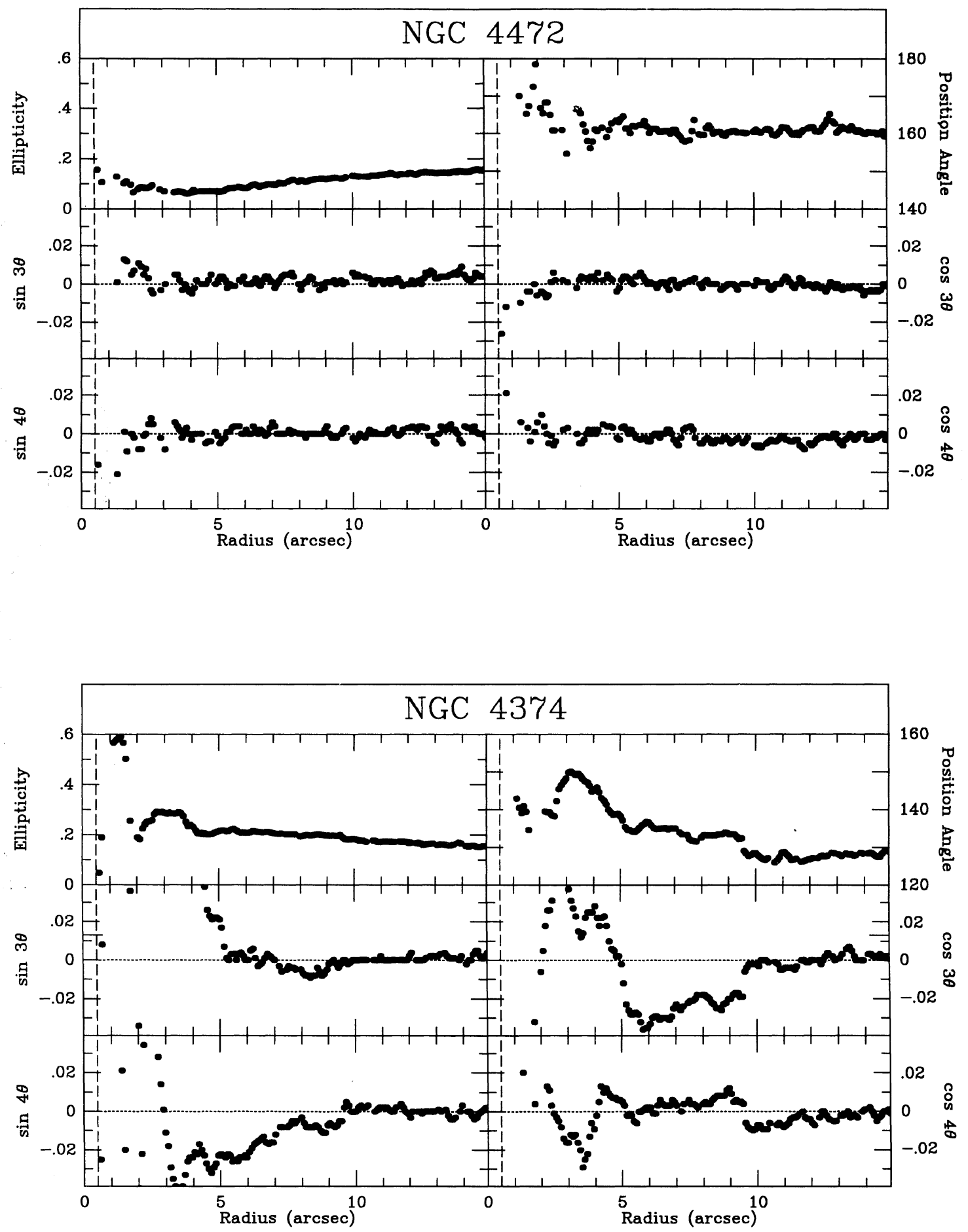

FIG. 6. Isophotal parameters as a function of semimajor axis length as derived from the Lucy deconvolved images of our sample of 13 Virgo ellipticals. Figure 6(n) shows the differences between the isophotal shape parameters derived from the Lucy deconvolved image of NGC 4342 and those found from the Fourier filtered image. Note that even though the isophotal structure of NGC 4342 is very complex $(\mathrm{m})$, the differences are very small. 

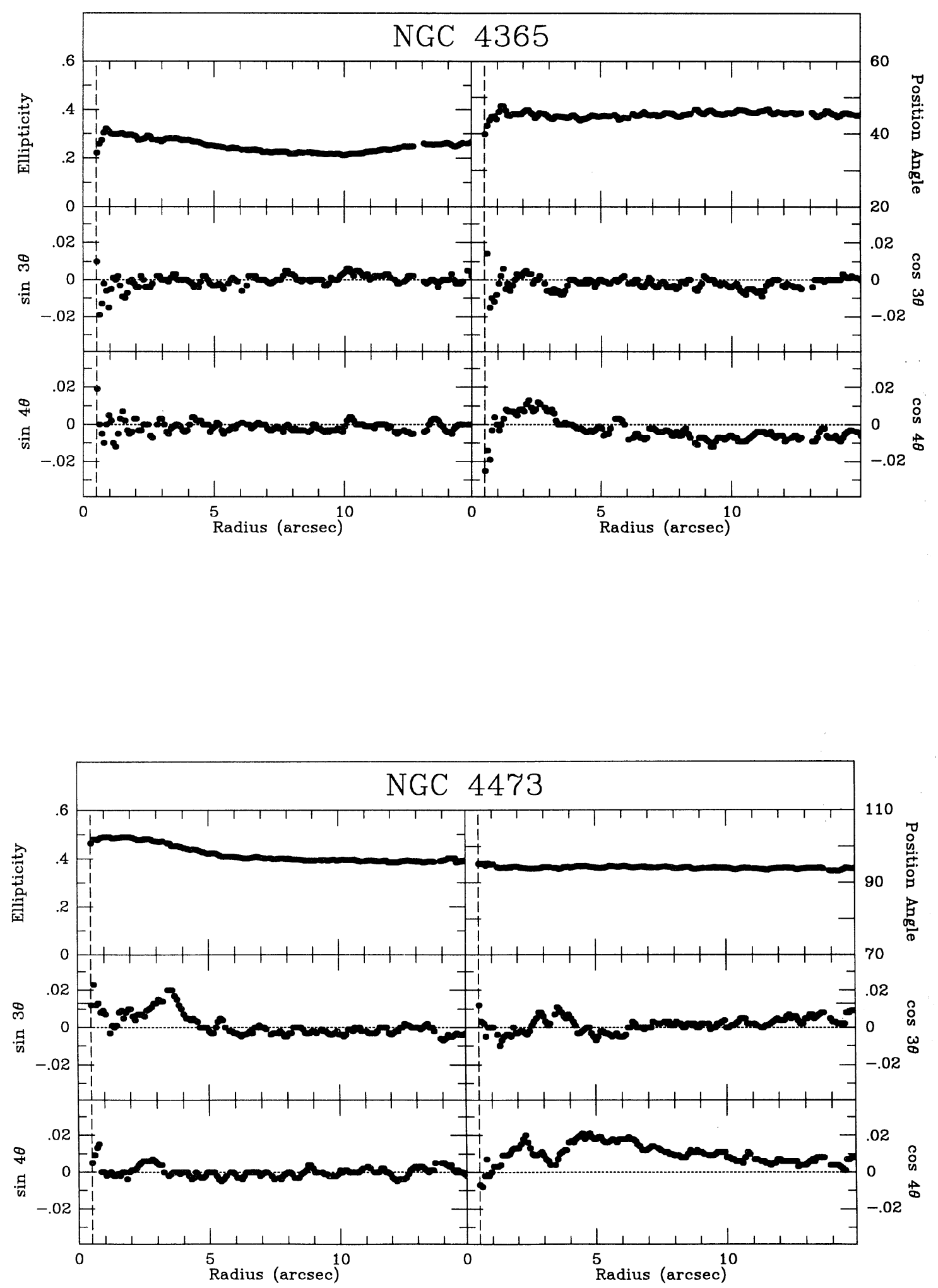

FIG. 6. (continued) 

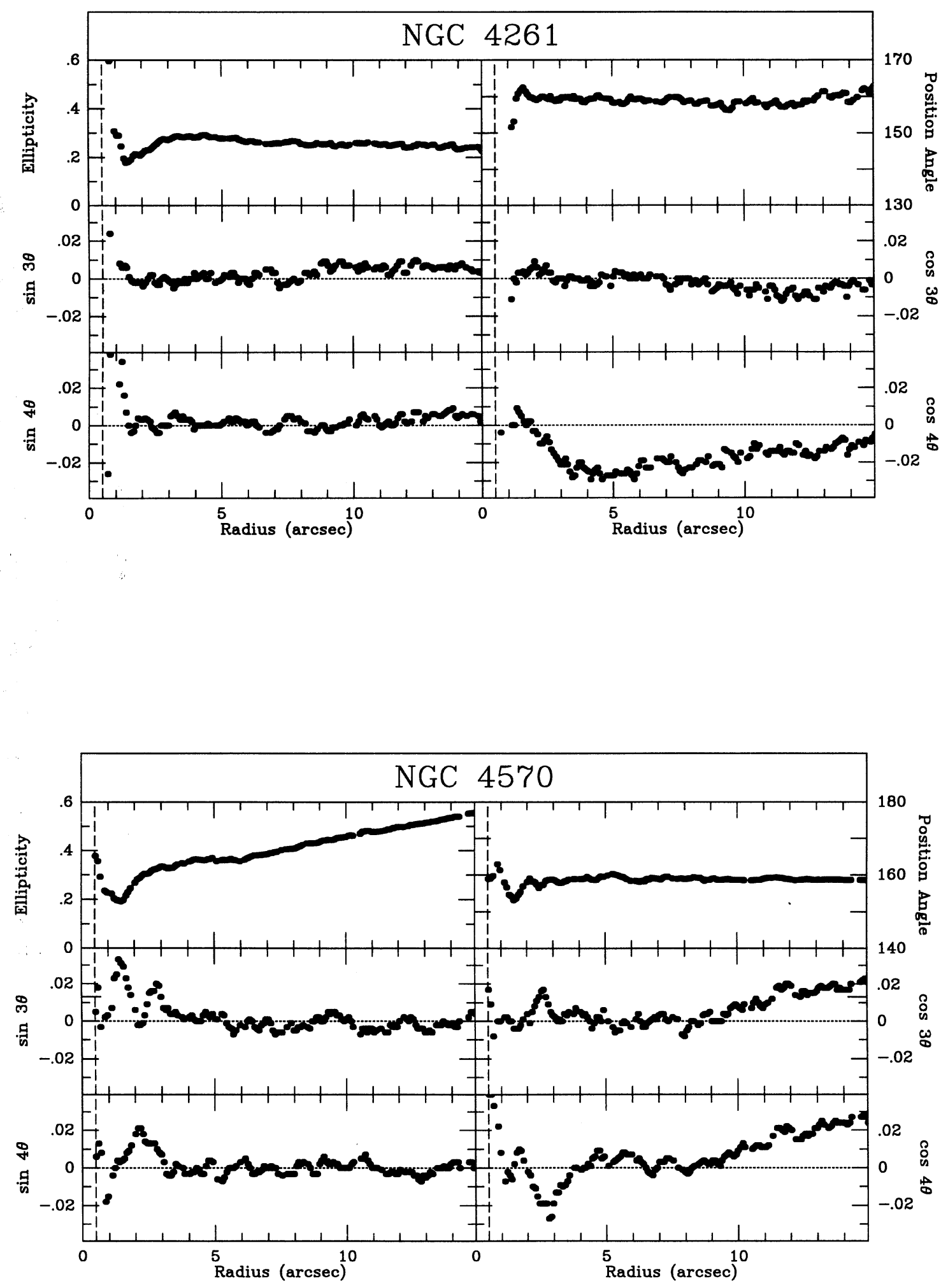

FIG. 6. (continued) 

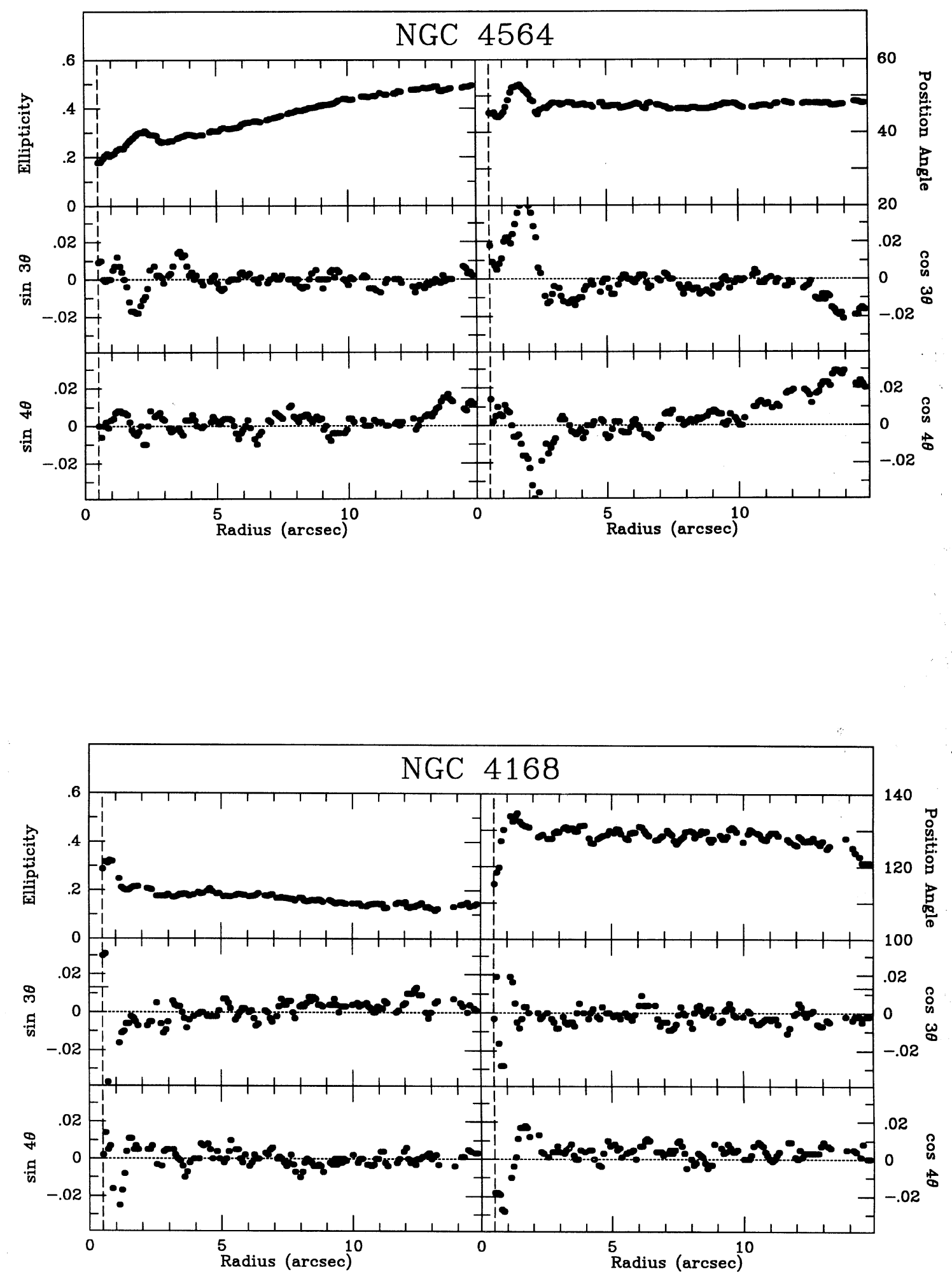

Fig. 6. (continued) 

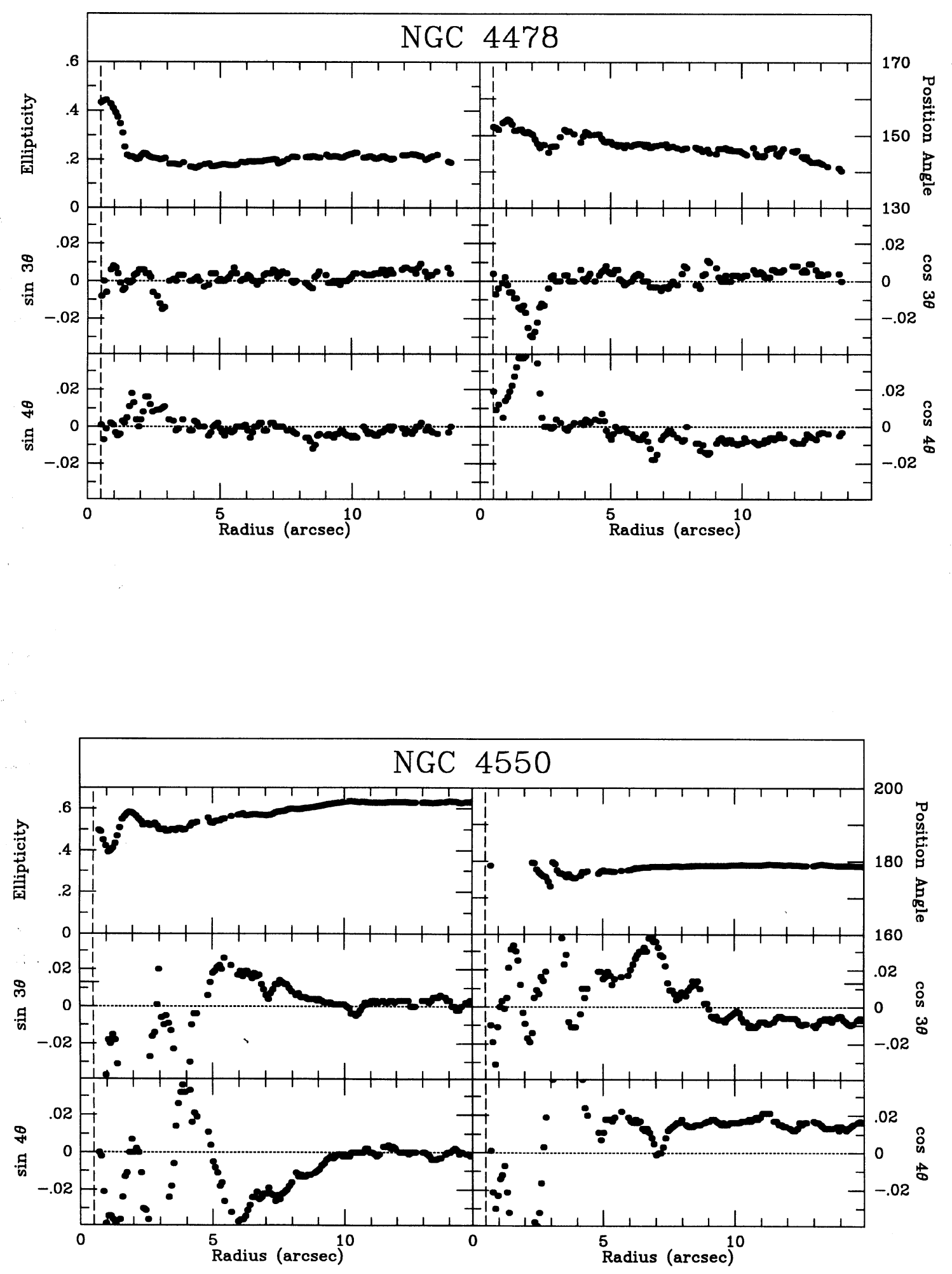

Fig. 6. (continued) 

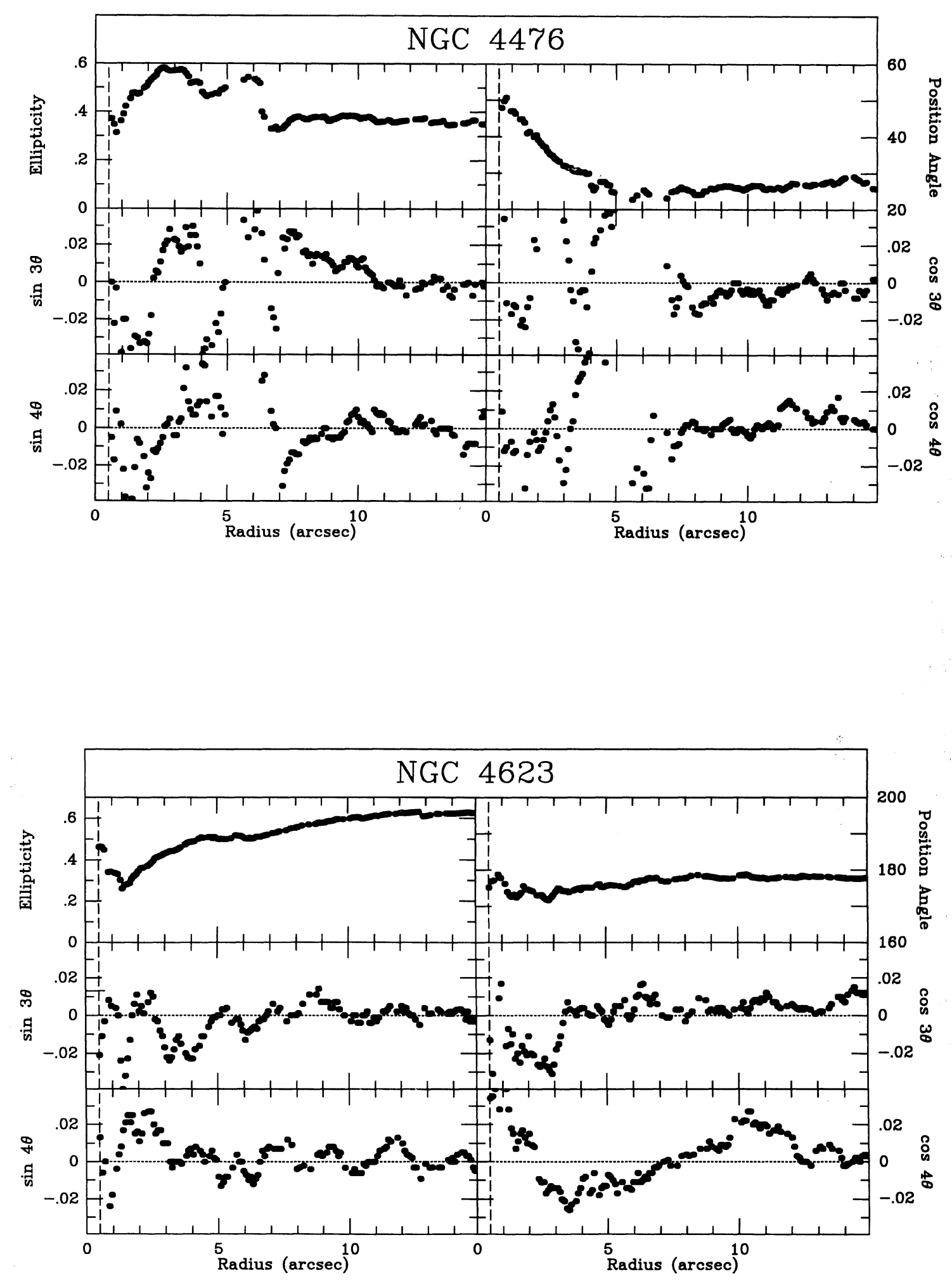

Fig. 6. (continued) 

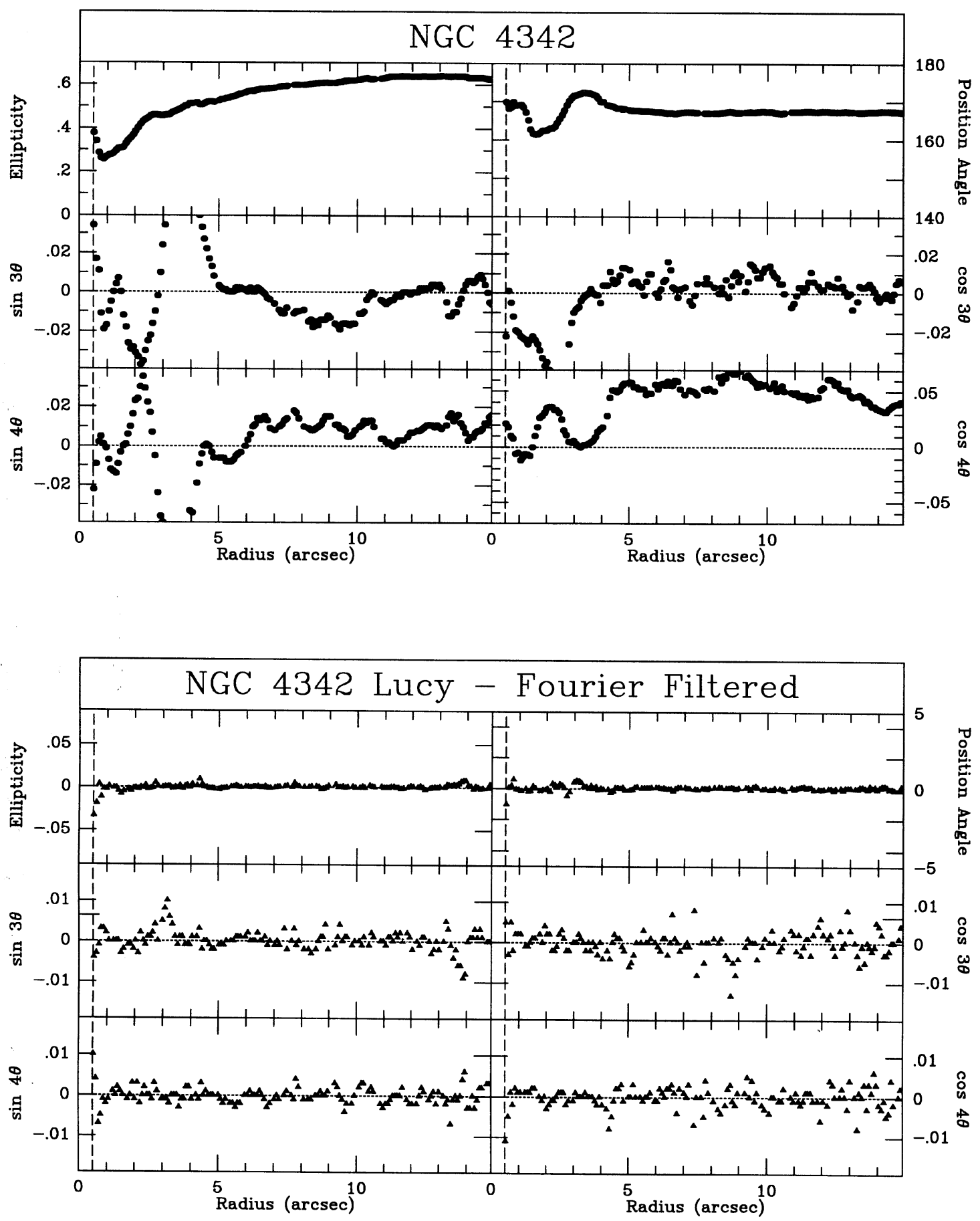

FIG. 6. (continued)

to 0.5 at $a=0.5$; the position angle remains fairly constant. These results are consistent with those found by Bender et al. (1988). The isophotes are disky at all radii. Between $2^{\prime \prime}$ and $4 "$ the higher order terms show small irregularities, that might indicate dust, although no gas and/or dust has been previously detected (Roberts et al. 1991, and see also Sec. 3.6).

3.4.5 NGC 4261 (3C 270)

The giant elliptical NGC 4261 is extensively discussed by Jaffe et al. (1993, 1994a). The center of NGC 4261 harbors a 
TABLE 1. Global isophotal parameters.

\begin{tabular}{|c|c|c|c|c|c|}
\hline $\begin{array}{c}\text { Galaxy } \\
\text { (1) }\end{array}$ & $\begin{array}{l}\langle\epsilon\rangle \\
(2)\end{array}$ & $\begin{array}{l}\langle\theta\rangle \\
(3)\end{array}$ & $\begin{array}{c}\langle\cos (4 \theta)\rangle \\
(4)\end{array}$ & $\begin{array}{c}\left\langle\frac{d \epsilon}{d r}\right\rangle \\
(5)\end{array}$ & $\begin{array}{c}\left\langle\frac{d \theta}{d r}\right\rangle \\
(6)\end{array}$ \\
\hline NGC 4472 & 0.122 & 160.9 & $-0.23 \pm 0.02$ & $+0.008 \pm 0.002$ & $0.4 \pm 0.7$ \\
\hline NGC 4374 & 0.187 & 130.9 & $-0.03 \pm 0.05$ & $-0.006 \pm 0.002$ & $1.1 \pm 0.5$ \\
\hline NGC 4473 & 0.396 & 94.0 & $+0.95 \pm 0.04$ & $-0.003 \pm 0.002$ & $0.0 \pm 0.1$ \\
\hline NGC 4261 & 0.255 & 158.9 & $-1.70 \pm 0.06$ & $-0.007 \pm 0.004$ & $0.6 \pm 0.5$ \\
\hline NGC 4570 & 0.454 & 158.9 & $+1.08 \pm 0.19$ & $+0.020 \pm 0.002$ & $0.1 \pm 0.1$ \\
\hline NGC 4478 & 0.204 & 145.8 & $-0.70 \pm 0.04$ & $+0.002 \pm 0.004$ & $1.1 \pm 0.5$ \\
\hline NGC 4550 & 0.608 & 178.6 & $+1.49 \pm 0.08$ & $+0.010 \pm 0.002$ & $0.09 \pm 0.07$ \\
\hline NGC 4476 & 0.376 & 25.4 & $+0.40 \pm 0.11$ & $-0.02 \pm 0.01$ & $0 \pm 1$ \\
\hline NGC 4623 & 0.578 & 177.6 & $+0.40 \pm 0.12$ & $+0.011 \pm 0.003$ & $0.3 \pm 0.2$ \\
\hline NGC 4342 & 0.610 & 167.4 & $+5.12 \pm 0.19$ & $+0.010 \pm 0.002$ & $0.08 \pm 0.06$ \\
\hline
\end{tabular}

Notes: The global isophotal parameters of the 13 galaxies in our sample, averaged over the radial range between $5^{\prime \prime}$ and $15^{\prime \prime}$. Column (1) gives the NGC number of the galaxy. Column (2) presents the average ellipticity, while the average position angle is in column (3) and the average $\cos (4 \theta)$-term in column (4). Columns (5) and (6) present the average gradients in the ellipticity and position angle profiles respectively.

sharply defined, elliptical dust disk, with axes of 1 ". $71 \times 0$ ".74. The disk precludes determination of the isophotal parameters for $a<1$ ". 1 , and induces large gradients at $r \leq 1$ ". 5 . At larger radii, the strongly negative $\cos (4 \theta)$ terms, indicate very boxy isophotes, consistent with previous studies (Bender et al. 1988; Peletier et al. 1990).

\subsubsection{NGC 4570}

NGC 4570 shows a strong decrease in ellipticity, running from 0.55 at $15^{\prime \prime}$ to 0.2 at 1.4 after which the ellipticity increases again to $\epsilon \approx 0.35$ at 0.5 . The position angle remains fairly constant. The $\cos 4 \theta$ terms shows remarkable behavior. Going outward, the isophotes start slightly disky, become boxy around $3^{\prime \prime}$ and become disky again at larger radii. The residual image [Fig. 7(a)] clearly reveals a disky structure of $\sim 2$.8 in diameter, aligned with the main body of the galaxy. The diskiness of the isophotes outside $9^{\prime \prime}$ is also due to a disk (see Sec. 3.7), and we therefore conclude that NGC 4570 closely resembles a lenticular (as defined by van den Bergh 1990), harboring a small nuclear disk in addition to its main disk. The small irregular variations of order $3 \%$ in the $3 \theta$ profile for $0.5<a<3$ " may indicate faint dust patches at these radii.

\subsubsection{NGC 4564}

NGC 4564 is highly elongated in the outer parts. The ellipticity reaches a maximum of $\sim 0.6$ between $30^{\prime \prime}$ and $40^{\prime \prime}$ (Bender et al. 1988; Caon et al. 1990). Further out the ellipticity decreases again. We find that the ellipticity decreases to 0.16 at 0.5 . Outside $10^{\prime \prime}$ the isophotes are disky (consistent with Bender et al. 1988). This behavior of the ellipticity profile is characteristic for a S0 (Michard 1984, cf. our discussions of NGC 4342, NGC 4570, and NGC 4623). Around $2 "$ the isophotal parameters are very irregular, but the residual map does not indicate the nature of the distortion. Gas and/or dust might be responsible although again Roberts et al. (1991) find no indication for either.

\subsubsection{NGC 4168}

This galaxy has a low surface brightness and a luminosity profile that flattens inside $\sim 2^{\prime \prime}$ (Paper III). Position angle and ellipticity are fairly constant from $2^{\prime \prime}$ to $15^{\prime \prime}$. Within this radial interval the higher order deviations are close to zero. Inside $\sim 2$ " all six parameters suddenly change. The residual map does not show a separate component in the inner $2^{\prime \prime}$, but the complex isophotal behavior here might be due to a small amount of dust as was previously argued by Ebneter et al. (1988). This would be consistent with the IRAS $100 \mu \mathrm{m}$ flux (see Paper I). Furthermore, [O III]-emission lines have been observed in this galaxy (Bettoni \& Buson 1987) as well as a radio continuum flux of $4 \mathrm{mJy}$ at $6 \mathrm{~cm}$ (Impey et al. 1986). NGC 4168 is thus a galaxy with a small amount of nuclear activity. This is the most plausible explanation for the "photometrically distinct nucleus" observed. At larger radii our results are in good agreement with Bender et al. (1988).

\subsubsection{NGC 4478}

This galaxy is, like NGC 4476, a companion of M87; its projected separation from M87 is 8.5. It is classified E2 in both RC2 and RSA. The lack of extended envelope indicates that NGC 4478 might be tidally truncated by M87. Prugniel et al. (1987) therefore classify NGC 4478 as a compact elliptical (cE). Previous studies on the photometry of NGC 4478 (Michard 1985; Prugniel et al. 1987; Bender et al. 1988; Peletier et al. 1990; Nieto et al. 1991a) all show a large isophote twist of $\sim 30^{\circ}$ between $30^{\prime \prime}$ and $60^{\prime \prime}$ (in agreement with what one might expect to find in the outer regions of a tidally truncated galaxy) plus a slow, constant change of position angle going inwards from $30^{\prime \prime}$. We find that this change of position angle continues down to 0.5 . Outside $6 "$ the isophotes are slightly boxy, consistent with previous studies. Inside $\sim 1.5$ we find a strong increase in ellipticity and diskiness with a negative $\cos 3 \theta$-term. The residual map [Fig. 7(b)] reveals a spot with slightly enhanced surface brightness at $1 . " 8(\sim 130 \mathrm{pc})$ from the center plus a string-like feature at the other side of the nucleus. This feature might represent ionized gas (none detected by Roberts et al. 1991), or a bright nuclear disk seen at an intermediate inclination 

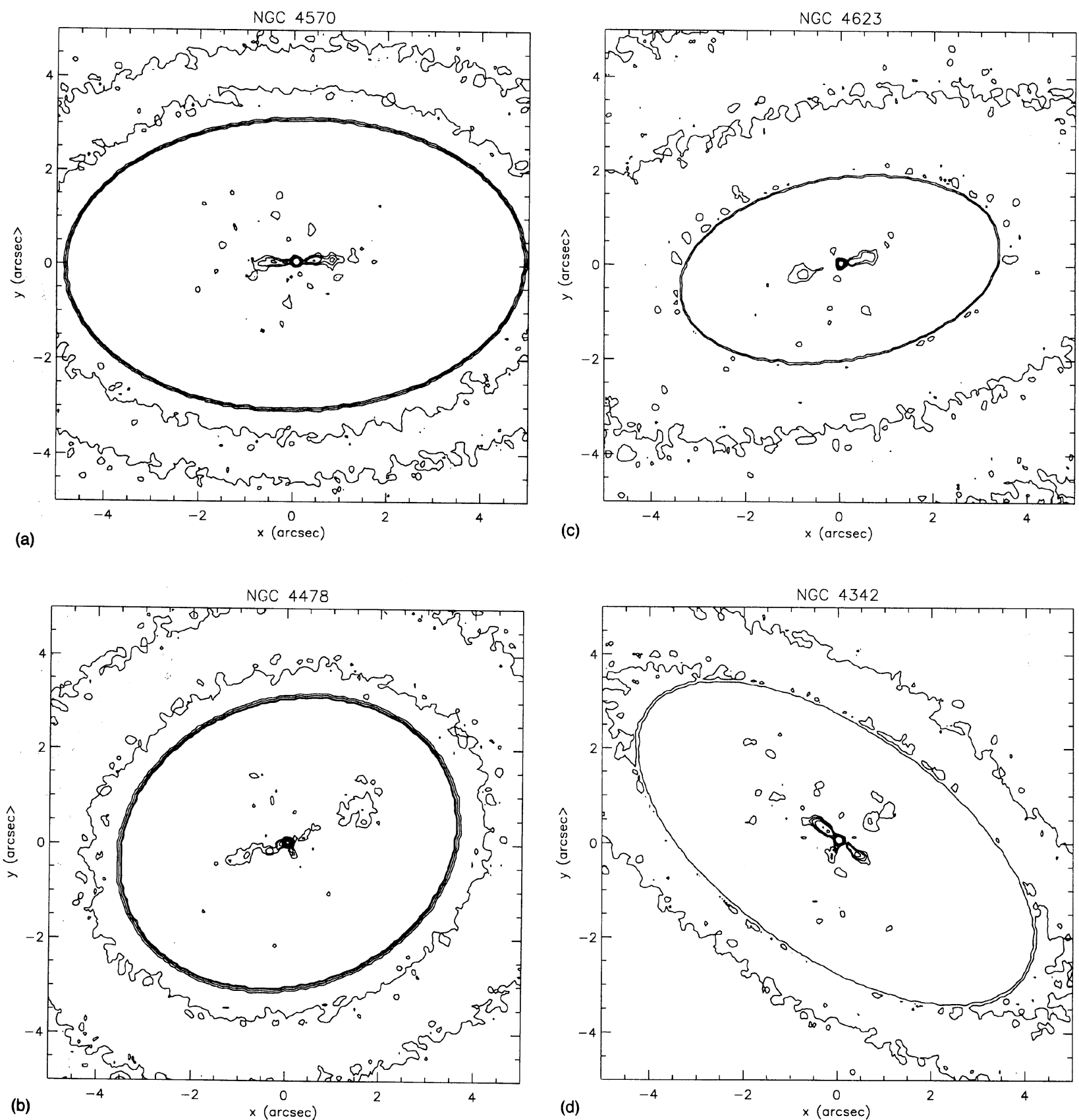

FiG. 7. Residual maps for a number of galaxies in our sample; (a) NGC 4570, (b) NGC 4478, (c) NGC 4623, and (d) NGC 4342. The maps are constructed by subtracting a model galaxy from the deconvolved image. The model has the same luminosity, ellipticity, and position angle profiles as the galaxy, but assumes the isophotes to be perfectly elliptical. These maps therefore show the third and fourth order residual structure. The models were not computed over the whole of the HST images. The sharp elliptical contours about 4" from the centers indicate the extent of the computed models.

angle. High resolution images in other colors and kinematic data are necessary to study this "photometrically distinct nucleus" in more detail.

\subsubsection{NGC 4550}

NGC 4550, is classified SBO in the RC2 and E7/SO1(7) in the RSA. It has two remarkably similar, co-spatial, counter-rotating stellar disks (Rubin et al. 1992; Rix et al. 1992). Filamentary dust in the inner region (see Paper I), distorts the isophotes at $a \leqslant 9^{\prime \prime}$. Outside this radius the isophotes are disky, consistent with Bender et al. (1988), and with the presence of the two disks.

\subsubsection{NGC 4476}

NGC 4476 is the second low luminosity companion of M87. Its lies 12' (projected) from M87 and $5^{\prime}$ from NGC 4478 (see above). NGC 4476 is classified SA(r)0 in the RC2, and E5pec(dust) in the RSA. This difference arises from the 


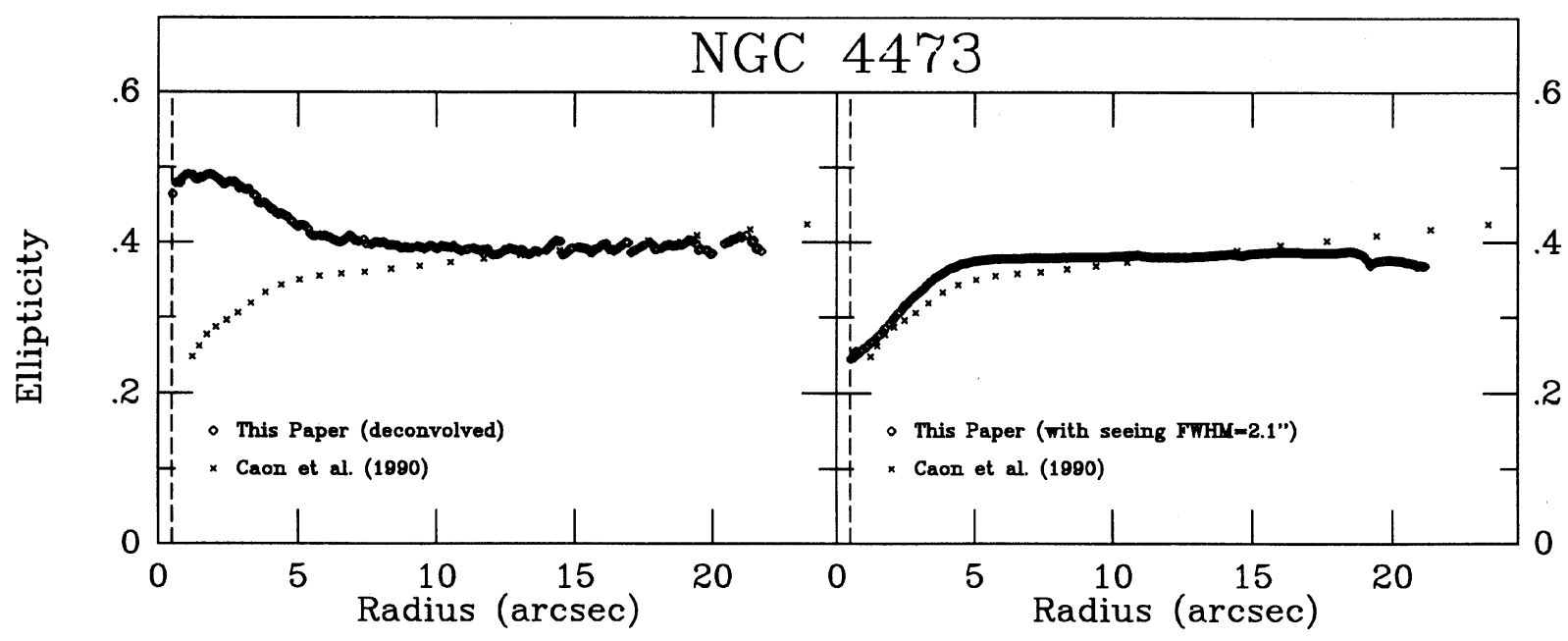

Fig. 8. The left panel compares our results with those of Caon et al. (1990) for NGC 4473. In the right panel we have convolved our Lucy deconvolved image with a Gaussian having a FWHM of 2"1, which is equal to the seeing reported by Caon et al. (1990). Clearly, the results after convolution are compatible.

presence of a dust ring in the central $\left(a \leqslant 7^{\prime \prime}\right)$ region, clearly visible in the HST image in Paper I. The ring disturbs the isophotal parameters inward of $7^{\prime \prime}$. Outside the ring we find a rather constant position angle of $\sim 25^{\circ}$, consistent with previous studies (Michard 1985; Prugniel et al. 1987; Caon et al. 1990), which all show that the position angle remains nearly constant out to $\sim 100^{\prime \prime}$.

\subsubsection{NGC 4623}

This galaxy resembles NGC 4570. It has a fairly constant position angle, an ellipticity decreasing inward with a sudden increase for $a<1.4$, and isophotes that are disky outside, boxy between $2^{\prime \prime}$ and $7^{\prime \prime}$ and disky again for $a \leqslant 2$ ". The residual map [Fig. 7(c)] reveals an inner disk with a diameter of $\sim 3^{\prime \prime}(210 \mathrm{pc})$ and aligned with the main body of the galaxy. Thus NGC 4623, like NGC 4570 , is a lenticular with both an inner and an outer disk. Variations in the $3 \theta$ profile at small radii may indicate dust. Taking the effects of seeing into account, these results are consistent with Nieto et al. (1991b).

\subsubsection{NGC 4342 (IC 3256)}

This highly elongated galaxy resembles an S0 more than an elliptical galaxy. It is classified E7 in the RSA Catalog but $\mathrm{S} 0$ in the RC2. Outside $5^{\prime \prime}$ the isophotes are extremely disky, and the position angle and ellipticity are constant. These results are consistent with Nieto et al. (1991b), but because of their bad seeing (FWHM=2.07), they did not detect the complex isophotal behavior inside $\sim 5^{\prime \prime}$. Although no dust or gas have been detected (Roberts et al. 1991), the HST image clearly shows two dust patches around $1 "-5^{\prime \prime}$ (Paper I). The residual image [Fig. 7(d)] shows a disk in the center of NGC 4342 , that is responsible for the rise in ellipticity and the sudden increase of the $\cos 4 \theta$-term at $a<0$." 8 .

\subsection{Comparison with Literature}

As explained in Sec. 3.2 we can accurately determine the isophotal shapes of ellipticals down to 0.5 , which is about one order of magnitude smaller than for Earth-based photometry. Seeing circularizes the isophotes in the inner regions and reduces the amplitudes of the higher order terms that indicate deviations from perfect ellipticity. We compared our results to several previous isophotal studies of the galaxies in our sample and found a very nice consistency. However, some differences were found which appear to be due to ground-based seeing. To test this we convolved our deconvolved images with a Gaussian having the same FWHM as the seeing of the ground-based studies to see if this interpretation is correct. Figure 8 shows these results for NGC 4473, when compared to the results of Caon et al. (1990). We found a much higher ellipticity inside $10^{\prime \prime}$, but when convolved with their seeing (FWHM=2"1) the results are consistent with Caon et al.. We still find an ellipticity which is slightly larger (approximately by $10 \%$ ), but we modeled the seeing with a perfect Gaussian, whereas this is not necessarily true. If the PSF has some exponential wings, for example, this will have a similar effect as a larger FWHM. We found the results for the other galaxies to be similar to those of NGC 4473.

\subsection{Dust}

As was shown in Paper I, we found clear evidence for dust in five galaxies (NGC 4261, NGC 4342, NGC 4374, NGC 4476, and NGC 4550). In general, dust will disturb the isophotes, which can result, for example, in large isophote twists (e.g., NGC 4476). If small dust patches or filaments are present, they will only be visible in the higher order terms of the Fourier expansion of the surface brightness. Peletier et al. (1990) and Goudfrooij et al. (1994) have shown that in particular the $3 \theta$ terms are sensitive indicators of the presence of dust. The five clearly dusty galaxies in fact show 
such irregularities. In Paper I we also noted faint dust patches in four additional galaxies: NGC 4472, NGC 4564, NGC 4186, and NGC 4623. Irregularities in the $3 \theta$ profiles can be seen in all these cases (cf. Fig. 6 and Sec. 3.4). In addition similar irregularities, probably indicating dust, are seen in NGC 4570 and NGC 4473.

\subsection{Stellar Nuclear Disks}

Although all galaxies in our sample seem to have their own peculiarities in their central regions, three galaxies have very similar isophotal structure. These galaxies (NGC 4342, NGC 4570, and NGC 4623) are very flattened, show a positive ellipticity gradient, have disky isophotes outside $5^{\prime \prime}-10^{\prime \prime}$, have a constant position angle, probably have small dust patches and, most surprisingly, harbor a small nuclear disk approximately $150 \mathrm{pc}$ in diameter. In all cases this nuclear disk is aligned with the main body of the galaxy. The diskiness of the isophotes, and the high ellipticity with a positive gradient outside $\sim 5^{\prime \prime}$ is most likely due to the an outer disk (or ring) component. In the region between the two disks there is a gap where the isophotes show a negative $\cos (4 \theta)$ term, suggesting that the bulge is boxy. The nuclear disks are found to extend $\sim 0.3$ along the minor axis in all three cases. Therefore, if we assume that the disks are intrinsically circular, the inclination angle of all three galaxies exceed $80^{\circ}$. Furthermore, we can conclude that the thickness of the nuclear disks is less than $25 \mathrm{pc}$.

The nuclear disks show up most clearly in the residual maps, but the outer disk component is hardly visible. This is due to the fact that the residual image is constructed by subtracting a model image that has the same luminosity and ellipticity profile as the real image, but is taken to have perfectly elliptical isophotes. Therefore, only the $\sin (n \theta)$ and $\cos (n \theta)(n>2)$ signatures of the disks show up in the residual map. These are only higher order effects and can therefore be rather small. We therefore tried another method of constructing a residual map for one of the galaxies in our sample that harbors an inner disk, NGC 4570. As is shown in Paper III, the non-disky ellipticals have luminosity profiles that can accurately be described by a double power-law in the inner 15 ". We assume that the ellipsoidal "bulge"component of the disky ellipticals has a similar doublepower law luminosity profile, which is of the form

$$
I=I_{c} \times\left\{\frac{1}{2}\left(\frac{r}{r_{c}}\right)^{2 \beta_{1}} \times\left[1+\left(\frac{r}{r_{c}}\right)^{2\left(\beta_{2}-\beta_{1}\right)}\right]\right\}^{-1 / 2} .
$$

We have fitted this function along the minor axis of NGC 4570 , where we have neglected the inner $\sim 1^{\prime \prime}$, which is affected by the nuclear disk (even along the minor axis). We find a reasonable fit for $\beta_{1}=0.80, \beta_{2}=2.33$, and $r_{c}=1$ ". 63 . From Fig. 6(f) we estimate the ellipticity of the bulge to be 0.18 , while its isophotes are slightly boxy $\left(B_{4}=-0.01\right)$. We have constructed a model-bulge that has the best fitting double-power law luminosity profile [Eq. (5)]. The axis ratio is equal to 0.82 , while $A_{n}$ and $B_{n}$ are zero, except for $B_{4}=-0.01$. We subtracted this model-bulge from the $H S T$ image. The resulting residual map is presented in Fig. 9. One can clearly distinguish a thick outer disk, down to $\sim 2^{\prime \prime}$ and a

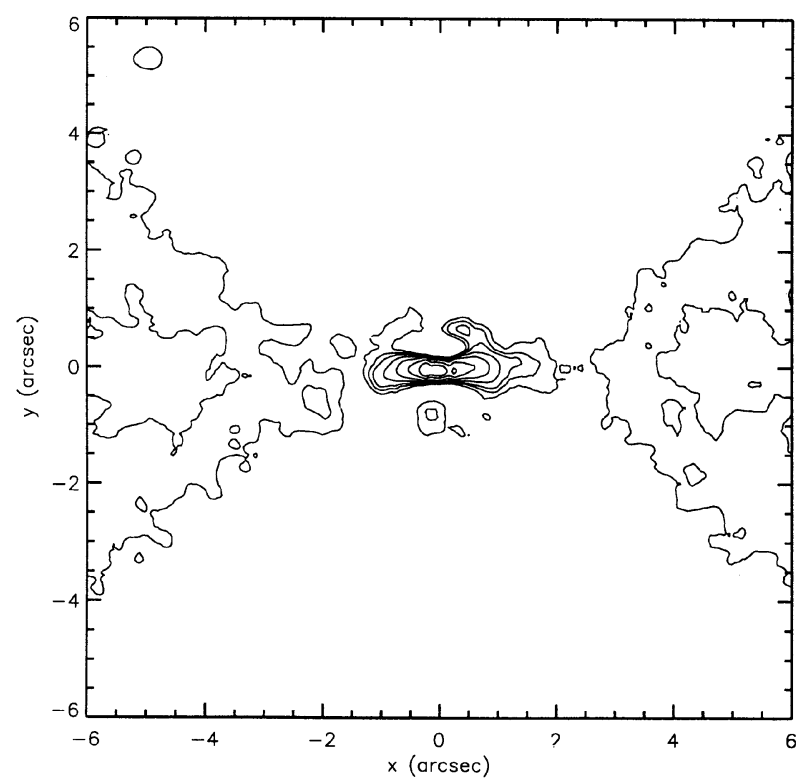

FIG. 9. The residual map of NGC 4570 after subtraction of a model of the bulge component. The model has a double power law luminosity profile, an ellipticity of 0.18 , and slightly boxy isophotes $\left(B_{4}=-0.01\right)$. Note the two separate disk components and the clear gap inbetween them.

much thinner disk inside $\sim 1.5$. Although the model of the bulge is somewhat ad hoc, this may reflect a general characteristic of the lenticulars, given the similarity of the isophotal structure of NGC 4342, NGC 4623, and NGC 4570.

\section{DISCUSSION AND CONCLUSIONS}

We have analyzed a homogeneous set of HST Planetary Camera images of Virgo cluster elliptical galaxies selected on the basis of their luminosity. Extensive testing on modelgalaxies has shown that we can accurately recover the isophotal parameters down to 0.5 . This is an order of magnitude better than possible for Earth-based photometry, which is severely limited by seeing. As was.shown in Sec. 3.2, seeing not only decreases the ellipticity, but also tends to decrease the absolute values of the Fourier coefficients $A_{n}$ and $B_{n}$.

We have deconvolved our data in two different ways (Fourier filtering and the Richardson-Lucy algorithm), and found that isophotal shape parameters of models and data are very similar for both methods of image restoration. The data have been rebinned to increase the signal-to-noise ratio and to be able to extend the analysis out to at least $15^{\prime \prime}$.

The HST images presented in Paper I clearly reveal dust, in different morphologies, in five of our 13 galaxies. Since the dust disturbs the isophotes, no strong conclusions can be drawn on the stellar component in the centers of these galaxies. From the isophotal analysis and a careful study of the images we find indications of dust in an additional six ellipticals. This brings the total number of ellipticals with dust to 11 out of 13 . This is a considerably larger fraction than the $\sim 30 \%-40 \%$ of ellipticals found to contain dust with groundbased imaging (e.g., Sadler \& Gerhard 1985). In seven of these galaxies dust had not been detected before. Observed 
from the ground dust had been detected in $31 \%$ of these ellipticals, consistent with the statistics previously mentioned. The fraction of ellipticals with dust more than doubles when the smaller features visible at $H S T$ resolution can be detected.

One of the most remarkable results we find from the isophotal analysis is that there seems to be a characteristic radius of $\sim 70-200 \mathrm{pc}$ (corresponding to $1^{\prime \prime}-3^{\prime \prime}$ at Virgo) in almost every galaxy. Outside this radius, the isophotal parameters are either constant or have a small gradient, while inside this radius, the isophotes often show rapid and irregular changes. It is intriguing that the characteristic radius we find present in the surface brightness profiles of these galaxies (see Paper III) is of the same order of magnitude.

Our observations show that the dichotomy between disky and non-disky elliptical galaxies, which was first recognized from studies on the $1-10 \mathrm{kpc}$ scale (e.g. Bender et al. 1989; Nieto et al. 1991c), clearly persists to the central $100 \mathrm{pc}$ region, which was heretofore not accessible for study. According to Table 1, all galaxies having $\langle\epsilon\rangle>0.4$ have a significant, positive ellipticity gradient plus disky isophotes. $(\langle\cos 4 \theta\rangle>0)$. This group of galaxies is also characterized by steep luminosity profiles (see Paper III). We interpret these observations as due to separate bulge and disk component(s). Therefore, these galaxies should preferably be described as lenticulars (Van den Bergh 1990). In three of these lenticulars, NGC 4342, NGC 4570, and NGC 4623, we found a stellar, disky component in the inner $\sim 1^{\prime \prime}$, aligned with the main body of the galaxy. These galaxies also show an outer disk, which vanishes around $5^{\prime \prime}-10^{\prime \prime}$. In the region between the two disks there is a gap where the isophotes are found to be slightly boxy, consistent with the finding of boxy bulges in spirals and S0s. Stellar disks embedded in the spheroid of an elliptical have been found in a number of other galaxies: e.g. NGC 2974 (Cinzano \& van der Marel 1993), NGC 3610 (Scorza \& Bender 1990) NGC 4125 (Capaccioli 1987), NGC 4660 (Rix \& White 1990), NGC 4697 (Carter 1987). The disks in these galaxies are much bigger than the $150 \mathrm{pc}$ nuclear disks found here. They might be similar though to the outer disk components that seems to be present in these three lenticulars. In the Sombrero galaxy, NGC 4594, several studies have revealed that the huge disk constitutes of two separate components; an outer and an inner disk (Burkhead 1986; Kormendy 1988). Emsellem et al. (1994) even find an indication for a third disk component in NGC 4594, namely a nuclear disk of $\sim 200 \mathrm{pc}$ in diameter. We therefore argue that the breaking up of the outer disk around $100 \mathrm{pc}$ might be a general characteristic of lenticulars, as well as the small and thin nuclear disk.
If nuclear disks are always present in this type of galaxies, we would also expect them in NGC 4550 and NGC 4623. In NGC 4550 the filamentary dust does not allow the stellar component of its central region to be studied in enough detail, while in NGC 4564 a possible nuclear disk might be undetected because of a too small inclination angle (note that NGC 4564 has the lowest mean ellipticity of this subsample). Rix \& White (1990) showed that a disk is undetectable for an inclination angle smaller than $53^{\circ}$. The extreme flatness of the nuclear disks indicate that dissipational processes must have been important in forming these galaxies. The distinct transition from nuclear to outer disk, with a clear gap in between, suggests that a dynamical instability at this radius determines this form. The thinness of the nuclear disks also suggest that these galaxies have never undergone collisions with other galaxies of significant mass (e.g., Ostriker 1990).

Finally we find that for those galaxies for which there is clear kinematic evidence for a decoupled core (NGC 4472 and NGC 4365), no anomalies are found in the central regions of these galaxies from the isophotal shape analysis. In fact NGC 4472 and NGC 4365 show the most regular isophotal behavior of all galaxies in our sample. Both are giant ellipticals and their luminosity profiles clearly flatten inward (Paper III). Many of the galaxies show some form of "photometrically distinct nucleus" caused by at least three different effects: the above mentioned nuclear disks, dust, and line emission from an active nucleus.

From this study we confirm that there are two subclasses of early-type galaxies (e.g., Bender et al. 1989; Nieto et al. 1991c). We also show that the nuclei of ellipticals often reveal interesting structures such as stellar disks, and dust in varies morphologies.

We would like to thank Roeland van der Marel, Robert Jedrzejewski, and Reinier Peletier for valuable discussions. F.C.vdB. acknowledges support from the Netherlands Foundation for Astronomical Research (ASTRON) (Grant No. 782-373-055) with financial aid from the Netherlands Organization for Scientific Research (NWO). He also thanks the hospitality of the Johns Hopkins University, where part of this work has been done. Partial financial support from NATO Grant No. 573373 is also acknowledged. H.F., L.F., and R.O.C. received partial support from NASA Grant No. GO-2607.01-87A. R.W.O. also received support from NASA Long Term Space Astrophysics Grant No. NAGW-2596.

\section{REFERENCES}

Baum, S. A., Heckman, T., Bridle, A., van Breugel, W., \& Miley, G. 1988, ApJS, 68, 643

Bender, R. 1988, A\&A, 202, L5

Bender, R., Döbereiner, S., \& Möllenhoff, C. 1988, A\&AS, 74, 385

Bender, R., Surma, P., Döbereiner, S., Möllenhoff, C., \& Madejsky, R. 1989,

A\&A, 217, 35

Bettoni, D., \& Buson, L. M. 1987, A\&AS, 67, 341
Burkhead, M. S. 1986, AJ, 91, 777

Burrows, C. J., Holtzman, J. A., Faber, S. M., Bely, P. Y., Hasan, M., Lynds, C. R., \& Schroeder, D. 1991, ApJ, 369, L21

Caon, N., Capaccioli, M., \& Rampazzo, R. 1990, A\&AS, 86, 429

Capaccioli, M. 1987, Structure and Dynamics of Elliptical Galaxies, IAU Symposium No. 127, edited by P. T. de Zeeuw (Reidel, Dordrecht)

Capaccioli, M., Held, E. V., \& Nieto, J.-L. 1987, AJ 94, 1519 
Carter, D. 1987, ApJ, 312, 514

Cinzano, P., \& van der Marel, R. P. 1993, Structure, Dynamics and Chemical Evolution of Elliptical Galaxies, in ESO Conference and Workshop Proceedings No. 45, edited by I. J. Danziger, W. W. Zeilinger, and K. Kjär, p. 105

Davies, R. L., \& Birkinshaw, M. 1988, ApJS, 68, 409

de Vaucouleurs, G., de Vaucouleurs, A., \& Corwin, H. C. 1976, Second Reference Catalogue of Bright Galaxies (University of Texas, Austin) ( $\mathrm{RC} 2)$

de Zeeuw, P. T., Peletier, R., \& Franx, M. 1986, MNRAS, 221, 1001

di Tullio, G. A. 1979, A\&AS, 37, 591

Ebneter, K., Djorgovski, S., \& Davis, M. 1988, AJ, 95, 422

Emsellem, E., Monnet, G., Bacon, R., \& Nieto, J.-L. 1994, A\&A (submitted)

Ferrarese, L., van den Bosch, F. C., Ford, H. C., Jaffe, W., \& O'Connell, R. W. 1994, AJ (submitted) (Paper III)

Franx, M., Illingworth, G. D., \& Heckman, T. 1989a, ApJ, 344, 613

Franx, M., Illingworth, G. D., \& Heckman, T. 1989b, AJ, 98, 538

Goudfrooij, P., Hansen, L., Jørgensen, H. E., Nørgaard-Nielsen, H. U., de

Jong, T., \& van den Hoek, L. B. 1994, A\&A (in press)

Hansen, L., Nørgaard-Nielsen, H. U., \& Jørgensen, H. E. 1985, A\&A, 149, 442

Impey, C. D., Wynn-Williams, C. G., Becklin, E. E. 1986, ApJ, 309, 572

Jaffe, W., Ford, H. C., Ferrarese, L., van den Bosch, F. C., \& O'Connell, R. W. 1993, Nature, 364, 213

Jaffe, W., Ford, H. C., Ferrarese, L., van den Bosch, F. C., \& O'Connell, R. W. $1994 \mathrm{a}$, in preparation

Jaffe, W., Ford, H. C., O'Connell, R. W., van den Bosch, F. C., \& Ferrarese, L. 1994b, AJ (submitted) (Paper I)

Jedrzejewski, R. I. 1987, MNRAS, 226, 747

Kennicut, R. C., \& Kent, S. M. 1983, AJ, 88, 1094

Kormendy, J. 1988, ApJ, 335, 40

Lucy, L. B. 1974, AJ, 74, 745
Michard, M. 1984, A\&A, 140, L39

Michard, M. 1985, A\&AS, 59, 205

Mihalas, D., \& Binney, J. J. 1981, Galactic Astronomy, 2nd Ed. (Freeman, San Francisco)

Miyamoto, M., \& Nagai, R. 1975, PASJ, 27, 533

Nieto, J.-L., Bender, R., Arnaud, J., \& Surma, P. 1991a, A\&A, 244, L25

Nieto, J.-L., Poulain, P., Davoust, E., \& Rosenblatt, P. 1991b, A\&A, 88, 559

Nieto, J.-L., Bender, R., \& Surma, P. 1991c, A\&A, 244, L37

Ostriker, J. P. 1990, in Evolution of the Universe of Galaxies, ASP Conf.

Ser., Vol. 10, edited by R. G. Kron (ASP, San Francisco), p. 25

Peletier, R. F., Davies, R. L., Illingworth, G. D., Davis, L. E., \& Cawson, M. 1990, AJ, 100, 1091

Prugniel, P., Nieto, J.-L., \& Simien, F. 1987, A\&A, 173, 49

Rix, H.-W., \& White, S. D. M. 1990, ApJ, 362, 52

Rix, H.-W., Franx, M., Fisher, D., \& Illingworth, G. D. 1992, ApJ, 400, L5

Roberts, M. S., Hogg, D. E., Bregman, J. L., Forman, W. R., \& Jones, C. 1991, ApJS, 75, 751

Rubin, V., Graham, J., \& Kenney, J. 1992, ApJ, 349, L9

Sadler, E. M., \& Gerhard, O. E. 1985, MNRAS, 214, 177

Sandage, A., \& Tammann, G. A. 1981, A Revised Shapley-Ames Catalogue of Bright Galaxies (Carnegie Institution of Washington, Washington, DC) (RSA)

Scorza, C., \& Bender R. 1990, A\&A, 235, 49

Snyder D. L. 1990, in The Restoration of HST Images and Spectra, edited by R. L. White and R. J. Allen (ST ScI, Baltimore), p. 56

Sparks, W. B., Ford, H. C., \& Kinney, A. 1993, ApJ, 413, 531

Surma, P. 1993, in ESO Conference and Workshop Proceedings No. 45, Structure, Dynamics and Chemical Evolution of Elliptical Galaxies, edited by I. J. Danziger, W. W. Zeilinger, and K. Kjär, p. 669

Van den Bergh, S. 1990, ApJ, 348, 57

Zeilinger, W. W., Møller, P., \& Stiavelli, M. 1993, MNRAS, 261, 175 